\title{
Research on Hydraulic Manifold Block Hole Routing Optimization Based on Improved Ant Colony Algorithm
}

\author{
Lile He, Ze Li, Zhijie Guo \\ School of Mechanical and Electrical Engineering, Xi'an University of Architecture and Technology, \\ Xi'an, 710055, China
}

Keywords: hydraulic manifold block; improved ant colony algorithm; hole routing; MATLAB.

\begin{abstract}
A collaborative evolutionary optimization method for the layout of hydraulic manifold blocks was presented according to its structure feature, an optimization design mathematic model of manifold blocks in the spatial layout was established, and it was solved based on the ant colony algorithm. Special mention was given here to demonstrate the improvement of current multiple ant colony collaborative evolutionary algorithms on transfer policy and rules of heuristic information which was made to Cooperative arrangement for hole routing. Finally, the simulation was carried out with MATLAB to verify the feasibility of the improved algorithm. The results of the simulation indicated that the improved algorithm was more available than the existing ant colony algorithms
\end{abstract}

\section{Introduction}

Hydraulic Manifold Blocks (HMB) which are the carrier to install many kinds of cartridge valve, the plate valves and other accessories, so they are the centre of the integrated hydraulic system. They assemble the control valve into "integral line" instead of the traditional external connection. They are characterized by compact structure, small vibration, and convenient installation and debugging. Nevertheless, the features of hole design are low efficiency and error-prone due to the nonstandard hydraulic system and the diversity of the hole connecting relationship with the manifold piece of internal hole often constitute a hole of interchange, dense complex network [1][2].

Manifold valve block structure is so complex that it is difficult to carry on the optimization design even with those CAD software presented in the papers available discussing on the same problem. HMB optimization is divided into two parts according to the design characteristics of $\mathrm{HMB}$, one is hydraulic components integrated block outer layout optimization, and the other one is internal route optimization. External layout optimization enable HMB to be the smallest with the premise of no interference between their components, while internal hole routing optimization has the advantage of the least cope hole of the single route and shortest total route with the premise of safety wall thickness[3]. Traditional hole routing optimization on simplex line is just the shortest path optimization, while the influences exerted by the hole order were ignored. The main objective of this study is to improve the algorithm for an efficient hole routing optimization, which not only realize the collaborative optimization of hole routing, but also guarantees algorithm a fast convergence rate.

\section{Space holes arrangement optimization model}

\section{Integrated Space Holes Arrangement Optimization Model}

The whole process of traditional hole connecting design is based on the experience or CAD, it is characterized by low efficiency and poor accuracy. Although the holes inside the HMB were various and complex in connecting relation, hole position parameters on the block is definite after the valve components layout on the HMB. The connecting design is proposed as follows:

Determine the depth of the hole on either side if the hole can be directly connected to the end.

Increase the hole number if the hole cannot be connected to the end directly to decrease the process hole number and length of route.

Verify the hole in the circumstances of various route layouts to prevent interference. 
In summing up what were described above can be derived the mathematical model for internal hole routing optimization design of HMB.

$$
\begin{cases}V_{k}=\left(F_{k}, X_{k}, Y_{k}, Z_{k}, R_{k}, L_{k}\right)^{T} & (k=1,2, \ldots, 5) \\ W_{t}=(O, A)^{T} & (t=1,2, \ldots, M) \\ O_{i}=\left(F_{i}, X_{i}, Y_{i}, Z_{i}, R_{i}, L_{i}\right) & (i=1,2, \ldots, P) \\ A_{j}=\left(f_{j}, X_{j}, y_{j}, Z_{j}, r_{j}, l_{j}\right) & (j=0,1, \ldots, N)\end{cases}
$$

Where $V_{k}$ and $W_{t}$ denote the hydraulic valve and route in HMB, respectively. $O_{i}$ and $A_{i}$ represent the working hole and process hole in hole, respectively. $F$ and $f$ are the parameters of the basal plane to connect two holes and to install the process hole, respectively. $R$ and $L$, denote the radius and depth of working hole, respectively. $r$ and $l$ describe the radius and depth of process hole, respectively. $X, Y, Z$ and $x, y, Z$ mean the coordinate of working hole and process hole, respectively.

Traditional connecting optimization design of HMB is to search two connecting hole with the shortest path and to meet the minimum hole length, using ant colony algorithm can achieve mutihole optimization design. According to (1), target function can be derived as follow:

$$
\left\{\begin{array}{l}
\min F(V, O, A)=\sum_{i=1}^{p} L_{i}+\sum_{j=0}^{N} l_{j} \\
\min G(V, O, A, M)=\sum_{k=1}^{M}\left(F_{k}\right)
\end{array}\right.
$$

Where $\min F(V, O, A)$ calculate the shortest path in every single connection. And $\min G(V, O, A, M)$ realizes the arrangement of all routes that can be connected at the same time, and the shortest path is obtained.

\section{Connection Solving of Space Hole Route}

The routing model space is the three-dimensional with a diagonal line through starting point and end point. There are a variety of stationary and obstacles those shape and locations are known in the space. The space of HMB can be divided into a $M \times L \times N$ grid nodes, each node identifies with an available coordinate $(x, y, z)$, the outside surface on HMB carrying hydraulic components were set as obstacles to be identified efficiently. The positioning holes are determined by the positions of hydraulic components when route in HMB, they are considered as a barrier due to its conflict with hole planning while in the process of route. It is necessary that the existing hole should be set as obstacle for identification owing to the routing order.

While with the instrument of improved ant colony algorithm to solve the problem presented above, a hole line corresponds to an ant route is made up of starting node, intermediate node and destination node in its population, the ants will search the shortest path in a manner of bypassing obstacles according to the identified obstacles in the process of solving the problem.

\section{Ant Colony Algorithm in Hole Connecting Problem}

Inspired by the ants foraging behaviour, a heuristic algorithm to figure out the combination Optimization problem is proposed by Colorni [4-5], namely Ant Colony Optimization algorithm (ACO). Evolutionary algorithm was improved by employing the biological coevolution mechanisms, which is aimed at the shortcomings of the genetic algorithm [6]. Compared with the traditional evolutionary algorithm, the impact exerted by the complex relationship between the environment and the individual's in the evolution is considered by collaborative evolutionary algorithm. A complex optimization problem can be decomposed into several interaction subsystem optimization problems by adopting the decomposition-coordination method. Co-evolution algorithm has achieved successful applications in either ship pipeline arrangement or path searching in intelligent air piping system [7-9]. The ant colony algorithm presented in the papers available is improved to speed up the algorithm's convergence and obtain more optimization results [10].

\section{Heuristic Information Rules}

Ants calculate the state transition probability by using the pheromone and heuristic information of each node while they are in the search process. The probability of motion that ants walk from starting point to destination is greater than that ants walk in an opposite direction, and they can bypass the barrier in a HMB hole planning with the ant colony algorithm. The heuristic information 
values of three nodes which are surrounded by the space that formed by current node and the end round are 2/9, and the heuristic information values of another three nodes outside space are 1/9. Further judgment is conducted according to the status value of each node at the same time, if its value is 1 , the heuristic information given by the cancellation and values it to 0 .

\section{The Pheromone Update}

It is significant that successful search can be realized by the ant colony algorithm through updating the pheromone, the pheromone is stored in the nodes of HMB model, a pheromone value is added to each node, the value of the pheromone represent the extent of attracting to ants, pheromone value on each nodes update after the ants pass, Therefore, the pheromone can update by using the following formula:

$$
\begin{aligned}
& \tau_{u, v, w}=(1-p) * \tau_{u, v, w}+\rho \Delta \tau_{u, v, w} \\
& \Delta \tau_{u, v, w}=\left\{\begin{array}{cl}
F \max (t), & (u, v, w) \in \text { global optimum } \\
0, & \text { etc }
\end{array}\right.
\end{aligned}
$$

Where $\rho$ and $1-\rho$ means the pheromone volatilization coefficient and pheromones residual factor, respectively. Because $1-\rho$ can be uniquely calculated by using $\rho$ according to (3) $\tau_{u, v, w}$ denotes the node pheromone value.

Since the results solved by the ant colony algorithm described above were out of accuracy, the improvement of heuristic and pheromone update strategy becomes more necessary to obtain the optimization results.

\section{Improvement of the Ant Colony Algorithm}

\section{Improvement of Heuristic Information Rules}

As heuristic information rules were described above used the normalized probability formulation, it is not necessary to use the form of fraction. This article uses the following rules to create inspiration: for the three nodes $\left(p_{1}, p_{2}, p_{3}\right)$ which are in the space formed by the current node and the end node, when move the three nodes from the current position to the direction of they moved before, the value of heuristic information rules is 30 , otherwise the value is 3 , and the rest of the value are 1 . This ensures that the ants move to the target's in a larger probability.

The motion should be kept as much as possible if the ant moved to the goal a moment before to achieve the desired effect that the ant both move to the goal and turn less. For the next mobile node, its heuristic information value is 0 if the ant is located in the path which has already walked or within the scope of the obstacle.

The following advantages are realized by improving the heuristic information rules presented above.

Ants have priority to move towards the goal.

Ants turn less.

Ants can bypass obstacles.

Ants move straight and avoid the phenomenon of circle or shock, make a reduction of total distance.

\section{Improvement of Pheromone Updating}

The accumulated pheromone on each node of its scene becomes more conducive for ants to find a better path with the iteration in progress. Not only do the adaptive values of optimization path have a decrease but also the largest fitness is also decreased in current global iteration. 


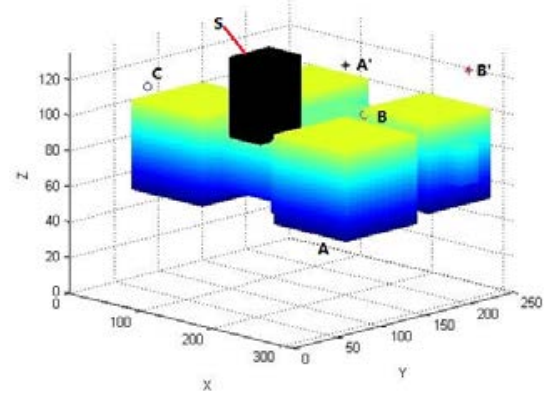

Fig.1 Obstacle model

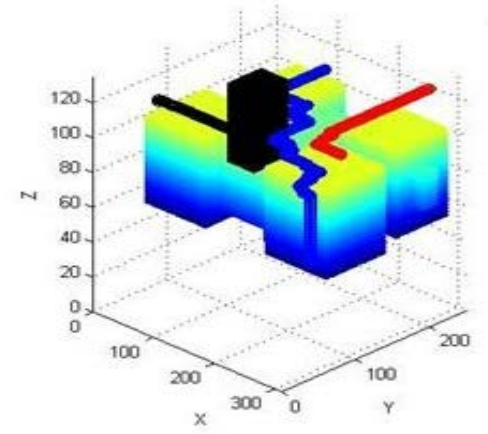

Fig.2 Layout results before improvement

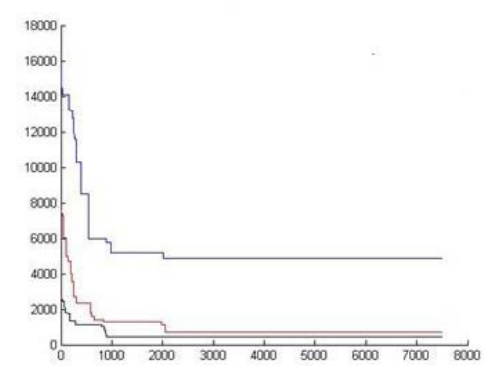

Fig.3 Adaptive value curves before improvement

The paths are more appropriate along with the increasing number of iterations path. In order to make an obvious increase of pheromone on optimization path, this paper adopts the following strategies: the global optimal path pheromones was found based on the strategy that global pheromone volatilize at each end of the iteration, the formula is presented as follows:

$$
\begin{aligned}
& \tau_{u, v, w}(t+1)=\Delta \tau_{u, v, w}(t)+(1-\rho) * \gamma / F_{\text {best }} \\
& \Delta \tau_{u, v, w}=\rho * \tau_{u, v, w}
\end{aligned}
$$

Where the $\gamma$ is the fixed coefficient, the value is 10000 in this paper. And $F_{\text {best }}$ represents the optimal solution adapted to the value.

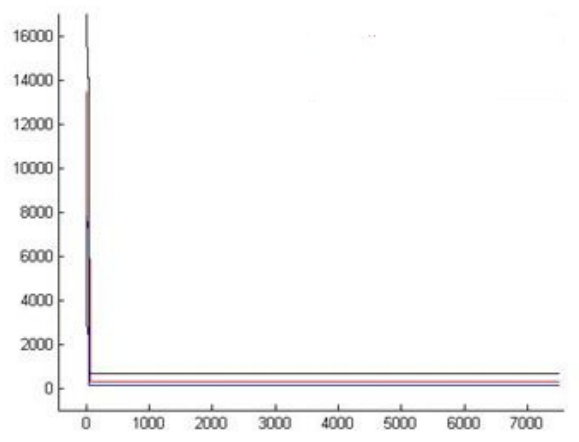

Fig.4 Adaptive value curves after improvement 


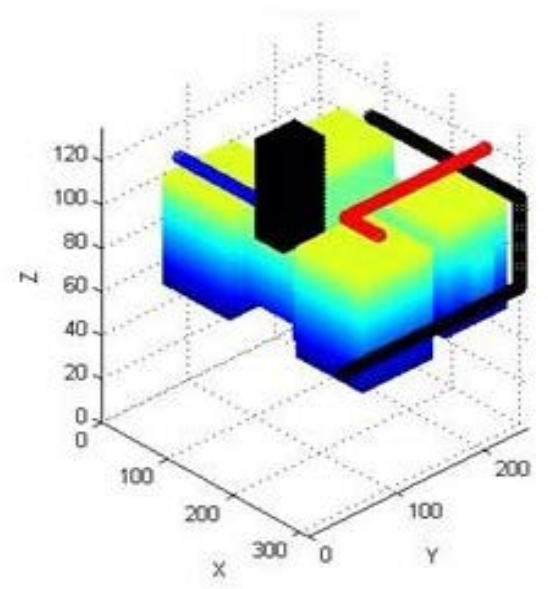

Fig.5 Layout results after improvement

This simulation is based on routing three groups inner holes in HMB, the results is verified by using MATLAB simulation algorithm. The model of environment of integrated three-dimensional space was established, and the obstacles treatment was employed to HMB and holes ready to route. As shown in Fig. 1, the obstacle model is distinguished to arrange to achieve a better accuracy, where $S$ is the set point of C's safety, A ' is connected with A, B ' is connected with B, the thickness of the wall for hole is set to $5 \mathrm{~mm}$.

According to the experience, we can set the parameters as follow, $\mathrm{M}=50, \rho=0.5$, $\alpha=1, \beta=2, q_{0}=0.8, \lambda_{1}=1, \lambda_{2}=1, \mathrm{Q}=1, \mathrm{GEN}=150, \mathrm{M}$ means the scale of ant colony, $\rho$ denotes pheromone volatilization coefficient, $\alpha$ represents information

Stimulating factor, $\beta$ is expectation stimulating factor, $q_{0}$ means threshold, $\lambda_{1}, \lambda_{2}$ represent the weight of path length and holes number in the objective function, respectively. $\mathrm{Q}$ is residual pheromone, GEN denotes the maximum number of iterations.

\section{Conclusions}

In this paper, an optimization design mathematic model of manifold blocks in the spatial layout is established. According to the simulation, a better optimization of routing was obtained by using the improvement in $\mathrm{HMB}$, the shock phenomenon was principally avoided as much as possible, and the reduction of turning is realized obviously, the convergence speed of improved algorithm is faster than that original with the condition of the guarantee to get the global optimal solution. However, convergence and accuracy of the algorithm remain to be improved. For that means, the improved algorithm has the possibility of being used in practical applications, although it is still under improvement through testing in field conditions.

\section{Acknowledgements}

The research work was supported by Scientific Research Program Funded by Shaanxi Provincial Education Department under Grant No.14JK1410.

\section{References}

[1] Chunmei Wang, Zhou Shijiang, Hongmei Xia, Chen Airui. The Design of Hydraulic Integrated Valve Block [J]. Journal of Coal Mine Machinery, 2008, 29 (9) : pp.40-41.

[2] Shujun Tian, Li Li. Optimization Design of Hydraulic Manifold Blocks Based on the Computing Intelligence [J]. China Mechanical Engineering, 2003 (7) : pp.35-37.

[3] Zhankui Wang, Xiqu Chen, Hongwei Jiao. Optimization and Implementation of Hydraulic Manifold Blocks Based On Genetic Particle Swarm Optimization (Pso) [J]. Journal of Engineering Design, 2008, 15 (1) : pp.66-71 
[4] Colorni A,Dorigo M,Maniezzo V,et al. Distributed Optimization By Ant Colonies[C]. Proc of the ECAL91.Paris:ECAL,1991:134-142.

[5] Maniezzo V, Colorni A. The Ant System Applied to the Quadratic Assignment Problem[J].IEEE Transactions on Knowledge and Data Engineering,1999,11(5):pp.769-778.

[6] Rosen C D,Belew R K.New methods for competive coevolution[J]. Evolutionary Computation,1998, (5) :pp. 1-29.

[7] Xiaoning Fan, Yan Lin, Zhuoshang Ji. Marine Pipeline Parallel Layout Optimization Based On Multiple Ant Colony Development [J]. Journal Of Shanghai Jiaotong University, 2009 lancet (2) : pp.66-71

[8] Xiaoning Fan, Yan Lin,Zhuoshang Ji. The Ant Colony Optimization for Ship Pipe Route Design in 3D Space. The Sixth World Congress on Intelligent Control and Automization, 2006,( 4):pp.3103-3108

[9] Jiang Fan, Mei Ma, Xiaoguang Yang. The Intelligent Search of Pipeline System Based on Development Localization Algorithm [J]. Journal of Aerospace Power, 2004, 12 (5) :pp. 593-597.

[10] Huanbing Cheng. Research and Development on Parameterized CAD Optimal Design System of Cartridge Valve [D]. Nanjing University of Science and Technology, 2014. 